\title{
Dynamo Action in Evolved Stars
}

\author{
David F. Gray \\ Department of Astronomy University of Western Ontario \\ London, Ontario N6A 3K7, Canada
}

\begin{abstract}
Evolved stars tell us a great deal about dynamos. The granulation boundary shows us where solar-type convection begins. Since activity indicators also start at this boundary, it is a good bet that solar-type convection is an integral part of dynamo activity for all stars. The rotation boundary tells us where the magnetic fields of dynamos become effective in dissipating angular momentum, and rotation beyond the boundary tells us the limiting value needed for a dynamo to function. The observed uniqueness of rotation rates after the rotation boundary is crossed can be understood through the rotostat hypothesis. Quite apart from the reason for the unique rotation rate, its existence can be used to show that magnetic activity of giants is concentrated to the equatorial latitudes, as it is in the solar case. The coronal boundary in the H-R diagram is probably nothing more than a map of where rotation becomes too low to sustain dynamo activity.
\end{abstract}

\section{The granulation boundary}

The asymmetries of spectral lines betray the presence of granulation in stellar photospheres. The combination of brighter granules moving upward, producing blueward Doppler shifts, with darker material moving downward, producing redward Doppler shift, is the reason for the asymmetries. The line bisector, i.e., the locus of points through the midpoints of horizontal line segments bounded by the sides of the spectral line profile, has been used to specify the asymmetries. Solartype line asymmetries produce bisectors sweeping redward near the continuum. A series of observations across the H-R diagram show solar-type bisectors on the cool half only. Some other (stronger) velocity fields appear to exist in the photospheres of hot stars, and the slope and curvature of their bisectors is reversed from the solar case (Gray and Nagel, 1989; Gray, 1990). The boundary between these two domains, where the asymmetry disappears, is the granulation boundary. Figure 1. shows the position of the granulation boundary. It corresponds to the onset of deep-envelope convection. It also corresponds to the onset of activity indicators, and I refer you to Gray and Toner (1986) Fig. 5.

Stars waste no time starting up activity even though from the stellar interiors point of view convection zones near the boundary are very thin. Nevertheless, the granulation structure of the velocity fields has apparently been established. Whether it is the herding of field lines to granule boundaries, or the Coriolis force twisting of flux, or something else that is the critical element here I don't know, but whatever it is, it starts up promptly at the granulation boundary. It also 
turns off promptly when stars evolve across this boundary from cooler to hotter temperatures, as the more luminous stars do during their blue-loop evolution.

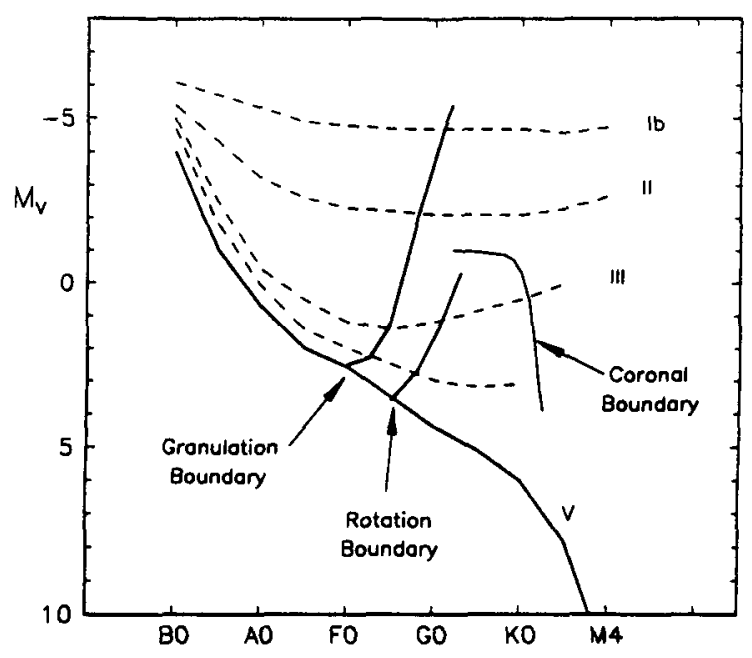

Fig. 1. The granulation boundary is clearly delineated by the line bisectors: left-leaning bisectors are seen on the left; right-leaning (solar type) on the right. Also shown here are the rotation boundary and the coronal boundary discussed below.

\section{The rotation boundary}

The drop of rotation along the main sequence through the early $F$ stars has been well documented over the years. The correspondence with the depth of the convective envelope and the increase of proxy-indicators of magnetic activity were pointed out a quarter of a century ago (Wilson, 1966; Kraft, 1967). Dwarfs give us a mass sequence. Giants, by contrast, give us something close to a constant-mass time sequence. Stars of Luminosity class III have masses too small to show blue loops, so increasing spectral type corresponds to increasing time. Figure 2. shows the precipitous drop in rotation between $\mathrm{G0}$ and $\mathrm{G} 3$ seen for single stars. A similar drop occurs for luminosity class IV (Gray and Nagar, 1985). The locus in the H-R diagram across which rotation suddenly drops is the rotation boundary shown in Fig. 1.

A dynamo-generated magnetic brake is almost certainly the cause of the sudden loss of angular momentum. Further, it must be a structural factor, as opposed to a time delay, because the rotational boundary parallels the granulation boundary even though the evolutionary times increase rapidly with lower luminosity. On the hot side of the boundary, the mean rotation is large and the distribution of rotation rates is Maxwellian. On the cool side of the boundary, the rotation is small, i.e. 6.1 $\mathrm{km} / \mathrm{s}$ at G3 III, and is a single-valued function of spectral type. My pet theory for understanding this is the Rotostat Hypothesis which says that dynamo activity ceases when rotation falls below a limiting value, but angular momentum brought 


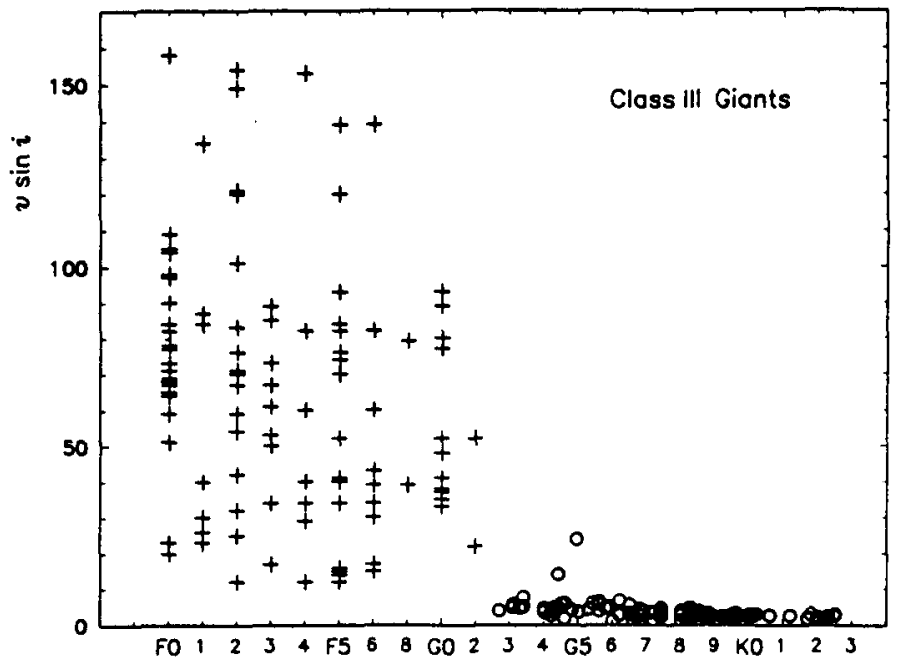

Fig. 2. The rotational break is shown for class III giants. (From Gray, 1989a.)

into the envelope as the lower boundary of the convection zone deepens with time keeps trying to spin up the star. As soon as the star rotates a bit faster than the limiting value, the dynamo comes to life, sets up its magnetic brake again, and rapidly reduces the rotation back to the limiting value. In this way rotation is a slave to the physics of the convection zone, and regulated by it.

\section{Equatorial activity}

We have precious little information on the latitude dependence of stellar activity, and it seems to me rather crucial information to have if we're going to understand which kinds of dynamos stars might be partial to. In the case of the sun, we naturally have detailed latitude mapping, and there we see tracers like spots localized to certain latitude bands. A glance at the "butterfly diagram" shows that sunspots prefer to stay within $\pm 45^{\circ}$ latitude, and in that sense they are concentrated toward the equatorial zone. It is true that the spatial resolution is so good for the sun that we can also see a narrow equatorial band where few sunspots occur. As a stellar astronomer, I ignore such fine detail.

The giants in question are those in the G3 to K2 III range where the rotation is a single-valued function of spectral type. Once we understand this fact, the mean value of $v \sin i$ at each spectral type can be converted to the actual $v$, under the assumption of random orientation of rotation axes, by multiplying by $4 / \pi$. Then each star's $v \sin i$ yields a $\sin i$ upon being divided by the now-known $v$. Pole-on stars have $\sin i=0$; equator-on unity.

Magnetic activity indicators can then be plotted as a function of orientation, as is done in Fig. 3 for the carbon emission. The carbon data is more recent and has smaller errors. But both plots show the same thing: stars seen near pole-on are 
weak emitters; those seen near equator-on are the strongest emitters. The curves in these diagrams show the geometrical factors, i.e., the amount of an equatorial belt that is visible as a function of axial orientation. Each curve is labeled with its latitude span, for example, $80^{\circ}$ is a belt running from $+40^{\circ}$ to $-40^{\circ}$ latitude.

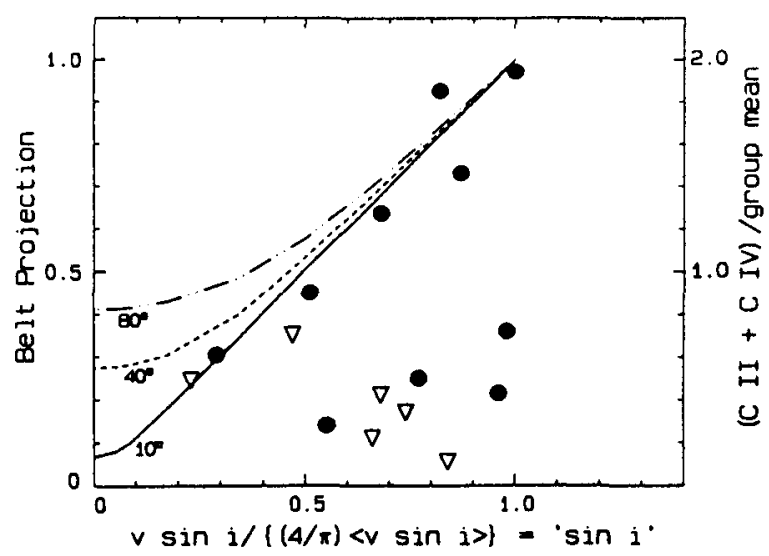

Fig. 3. Carbon emission (right ordinate) is shown as a function of axial orientation (computed $\sin i$ ). The left ordinate shows the fraction of a photosperic equatorial belt that is visible at any orientation. Each curve is labeled with the full latitude width of the belt. (From Gray, 1990.)

Some of the points in these figures lie well below the belt curves, but that is what we would expect if the strength of activity indicators varies with rotation phase, long-term cyclic pattern, and/or rotostat flickering. Axial orientation is one more variable contributing to the range of activity-indicator strength seen in otherwise homogeneous groups of stars.

Apparently normal giant stars have a liking for the the same dynamo patterns seen on the sun.

\section{The coronal boundary}

Indicators of coronae such as $\mathrm{x}$-rays show a domain in the H-R diagram bounded on the right and top, as shown in Fig. 1. Coronae are seen for stars "under" the boundary, but for the very luminous and the very cool stars, none of the usual coronal indicators appear (Linsky and Haisch, 1979; Ayres et al., 1981; Haisch and Simon, 1982). Explanations have been given for this in terms of physical structure of the outer atmosphere (e.g., Antiochos and Noci, 1986; Böhm-Vitense, 1987), but a simpler explanation lies with the rotation itself: star outside the coronal boundary rotate too slowly to sustain dynamos. Stars of high luminosity rotate below the dynamo limit because evolutionary expansion has increased their moment of interia sufficiently to make it so across the whole domain to the right of the granulation boundary. Stars to the right of the coronal boundary 
continue to struggle along at the dynamo limit until they reach spectral types $\approx$ K2 III. There their evolution carries them up the asymptotic branch where the track in the $\mathrm{H}-\mathrm{R}$ diagram changes from nearly horizontal to nearly vertical. This new tack involves a rapid increase in the moment of inertia, and the rotation dives below the dynamo limit, squelching the support of coronae.

Evolved stars clearly supply a wealth of information about dynamos. We need to connect it more explicitly to dynamo calculations.

To those whose thoughts may have drifted a bit, let me express it a different way:

\title{
Dynamos Keep Us Going in Circles
}

\author{
Dynamos, dynamos, friend or foe? \\ with all those equations, my head works too slow! \\ But of stellar activity give us a taste, \\ and these grand efforts won't go to waste. \\ Theory in one hand, data in the other,
} don't go and hide, but join hands with your brother.

Bring 'em together and turn up the heat.

We'll cook up some theories that will be hard to beat.

Put the pieces in place over the years, even though the slow pace brings us to tears.

On we struggle with truth our goal.

Cosmic detectives our chosen role.

Now magnetic fields string everything together, but their motions and antics change like the weather.

Twist 'em and turn 'em and wiggle 'em around.

Who knows what we're doing? Is any of it sound?

Well, look at the data; look where it's led.

We can tie it together by using our head.

The weakest example of many around,

the solar dynamo we continue to hound.

While other stars show us more dramatic effects,

essential evidence one sometimes neglects.

Evolved stars are special in what they do.

So watch carefully for clue upon clue.

Across that boundary of granulation, convection joins rotation to bring consummation.

Then rotation, once fast, is soon slowed down.

No more wild spinning or playing around!

To each spectral type the rotation's unique.

Doesn't this tell us something we seek?

You bet it does; in your heart it's felt.

Activity for giants comes round in a belt. 
Do dynamos cease or do they flicker

in that rotostat mode over which we dicker?

It's all good fun; don't sit there alone.

Join in with spirit; your heart's not a stone.

Dynamos are really a tricky beast.

It's obvious from this intellectual feast.

And so on to those questions you surely will ask,

making for this day the end to my task.

\section{References}

$$
\text { D.F.G. }
$$

Antiochos, S.K., Noci, G.: 1986, Astrophys. J. 301, 440

Ayres, T.R., Linsky, J.L., Vaiana, G.S., Golub, L., Rosner, R.: 1981, Astrophys. J. 250, 293

Böhm-Vitense, E.: 1987, Astrophys. J. 301, 297

Gray, D.F.: 1986, Highlights in Astronomy, Reidel, Dordrecht, ed. J.-P. Swings, p. 411

Gray, D.F.: 1988, Lectures on Spectral-Line Analysis: F, G, and K Stars, (The Publisher: Arva, Ontario)

Gray, D.F.: 1989a, Astrophys. J. 347, 1021

Gray, D.F.: 1989b, Publ. Astron. Soc. Pac. 101, 1126

Gray, D.F.: 1990, Publ. Astron. Soc. Pac., accepted

Gray, D.F., Nagar, P.: 1985, Astrophys. J. 298, 756

Gray, D.F., Nagel, T.: 1989, Astrophys. J. 341, 421

Gray, D.F., Toner, C.G.: 1986, Publ. Astron. Soc. Pac. 98, 499

Haisch, B.M., Simon, T.: 1982, Astrophys. J. 263, 252

Kraft, R.P.: 1967, Astrophys. J. 150, 551

Linsky, J.L., Haisch, B.: 1979, Astrophys. J. Letters 229, L27

Wilson, O.C.: 1966, Astrophys. J. 144, 695 